\section{Assessing the spatial extent of breast tumor intrinsic optical contrast using ultrasound and diffuse optical spectroscopy}

\author{
Ang Li, Jing Liu, Wendy Tanamai, Richard Kwong, \\ Albert E. Cerussi, and Bruce J. Tromberg* \\ University of California, Irvine, Beckman Laser Institute and \\ Medical Clinic, Laser Microbeam and Medical \\ Program, Irvine, California 92612
}

\begin{abstract}
Little is known about the relationship between anatomic and functional contrast derived from intrinsic optical signals. In order to address this relationship, finiteelement (FEM) forward simulations were compared to diffuse optical spectroscopy (DOS) reflectance measurements obtained from 10 breast tumor patients. Clinical ultrasound images were used to estimate anatomical tumor size and depth for the FEM simulations. Actual DOSmeasured tumor absorption could not be matched by forward model simulations when tumor size was constrained to match ultrasound dimensions. However, agreement was achieved when the lesion was viewed as a distribution of optical properties (i.e., an extended target). This result suggests that the spatial extent of optical contrast in breast tumors may be significantly greater than anatomical dimensions reported by standard imaging modalities. Analysis indicates that invasive breast tumors with anatomical dimensions of $1 \mathrm{~cm}$ may still be detectable at depths of $30 \mathrm{~mm}$ or more (the center of the lesion to the surface of tissue) using DOS in a reflectance geometry. () 2008 Society of Photo-Optical Instrumentation Engineers. [DOI: 10.1117/1.2937471]
\end{abstract}

Keywords: diffuse optical imaging (DOI); photon migration; ultrasound; breast cancer; near infrared.

Paper 07481LR received Dec. 14, 2007; revised manuscript received Feb. 25, 2008; accepted for publication Mar. 31, 2008; published online Jun. 20, 2008.

Diffuse optical spectroscopy (DOS) is an emerging diagnostic technique that quantitatively measures the concentrations of deoxy-hemoglobin, oxy-hemoglobin, water, and lipid in cm-thick tissues. ${ }^{2}$ In our experimental configuration, DOS samples a low number of spatial locations (i.e., a limited number of source-detector pairs) in a reflectance geometry with a large spectral bandwidth. In contrast, diffuse optical tomography (DOT) and diffuse optical imaging (DOI) typically sample a large number of spatial locations in planar and circular transmission geometries but with low spectral bandwidth. $^{1,2}$

Accurate reconstruction of heterogeneity coordinates and optical properties within an optically diffusive medium is a challenging problem. Because DOS typically uses only simple homogeneous models and a limited number of source-detector

*Tel: 1-949-824-4713; Fax: 949-824-8413 pairs, the measured optical properties of heterogeneities such as tumors can be a weighted average of target and background optical properties (i.e., absorption coefficient, $\mu_{a}$, and reduced scattering coefficient, $\mu_{s}^{\prime}$ ). The target is modeled as a spherical (or other shape) subsurface heterogeneity. The sphere represents a localized perturbation in optical properties relative to a "normal" and otherwise homogeneous background. Multispatial tomographic methods stratify the optical properties between target and background regions, usually at a few wavelengths. Significant artifacts have been eliminated in DOT by introducing a priori spectral information as well as spatial information from other imaging modalities. ${ }^{3-7}$

In this work, we used DOS to measure tissue optical properties within a 2-D spatial grid that covered the breast lesion (i.e., a DOS "gridscan"). We used standard forward modeling to determine the effect of $\mu_{a-h e t}$ and $\mu_{s-h e t}^{\prime}$ (i.e., $\mu_{a}$ and $\mu_{s}^{\prime}$ of the heterogeneity) on DOS without tomographic reconstructions. For simplicity, we present only a single frequencydomain wavelength, $658 \mathrm{~nm}$, and measurements across only one line (i.e., a "linescan") of $\mu_{a}$ within the 2-D grid. (We chose the line with the highest contrast). The raw DOSmeasured data (i.e., frequency-domain amplitude and phase) were calibrated and fit to a semi-infinite homogenous diffusion model in the least squares sense. ${ }^{1}$ We present a typical case in Fig. 1(a). This patient has a tumor of ultrasound (US) dimension $15 \times 12 \times 11 \mathrm{~mm}$ at a depth of $11 \mathrm{~mm}$. All DOS reflectance linescans were performed over the tumor region and included diseased and normal tissues. The linescan was based on a priori knowledge of the lesion location from standard US imaging. DOS linescans were taken in 10-mm steps with a $28 \mathrm{~mm}$ source-detector separation.

There was approximately a twofold difference between $\mu_{a-a v g}$ at the center and extreme linescan positions. ( $\mu_{a-a v g}$ is the recovered bulk absorption at the measurement point using a semi-infinite homogenous model.) This is consistent with our most recent 58 patient study of stage II/III malignant tumors. ${ }^{1}$ We fit the spatial distribution of $\mu_{a-a v g}$ values (i.e., the DOS linescan) in Fig. 1(a) to a Gaussian distribution. There is a large difference between the DOS full-width halfmaximum (FWHM) linescan $(26 \mathrm{~mm})$ and the US FWHM $(15 \mathrm{~mm})$. This discrepancy is due, in part, to intense multiple scattering. ${ }^{7}$

Using a breast lesion model based on a priori spatial information (i.e., size and depth) obtained by US, we employed a finite-element (FEM) forward solution (COMSOL Software, Palo Alto, California) to simulate frequency-domain amplitude and phase along a simulated linescan. The choice of target optical properties was based on four assumptions. First, the target lesion formed a well-defined perturbation, with a spatial extent equal to the US size and depth. Second, due to the optical property averaging, we assumed that the target optical absorption should be at least $2 \times$ the background. Further, the hemoglobin contrast is likely less than $5 \times$ the background based upon histological studies. ${ }^{8}$ Third, based upon our previous DOS clinical studies, we assumed that the scattering coefficients of the target and the background were $1.2 \mathrm{~mm}^{-1}$ and $1 \mathrm{~mm}^{-1}$, respectively. ${ }^{1}$ Fourth, we assumed that all regions satisfy the diffusion approximation, a standard assumption for similar problems.

We fit the simulated raw data to the semi-infinite homogenous diffusion model in exactly the same manner as the 


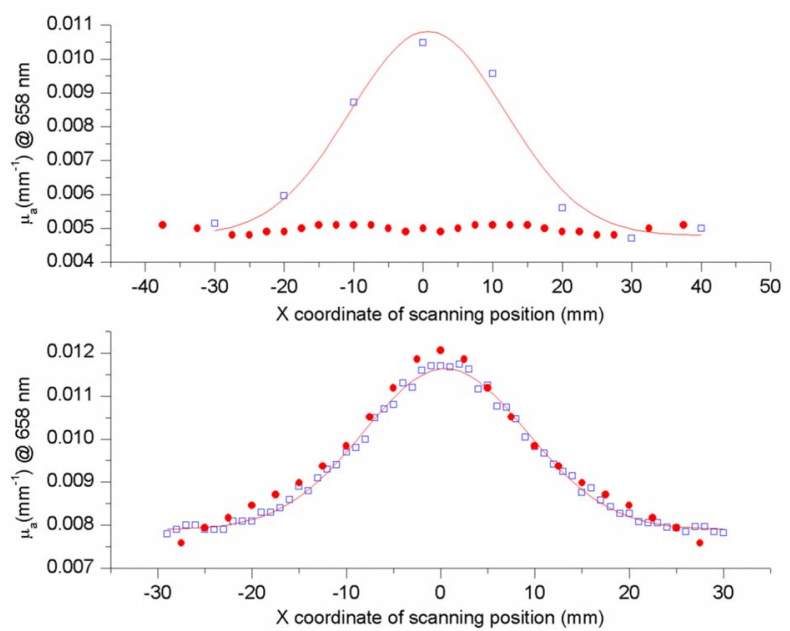

Fig. 1 Comparison of FEM forward simulation and DOS-measured linescans using a discrete target. The squares are $\mu_{\text {a-avg }}$ measured over the tumor (top) and phantom (bottom). The circles are $\mu_{\text {a-avg }}$ at each simulated linescan location using a well-confined breast tumor model. The line is a Gaussian fit to the measured linescan.

experimental data and compared simulated and measured linescans. We manually updated the tumor model in order to deduce the target optical property values that reproduced the measured experimental results. Figure 1(a) shows the simulation results using the US tumor size with a $\mu_{a-h e t}$ that is 5 $\times \mu_{a-b k g}$. There are large discrepancies between experimental (squares) and simulated (circles) linescans that cannot be explained by experimental or numerical noise. This result is difficult to understand given the lesion's relatively shallow depth and high optical contrast. A wide range of target absorption and scattering properties were tested, ranging up to $5 \times$ the background for $\mu_{a}$ and $2 \times$ the background for $\mu_{s}^{\prime}$. Increasing target volume by $50 \%$ had little effect on the results.

To test the validity of our model, we constructed a phantom consisting of a homogenous turbid silicone matrix (i.e., background) with a cylindrical cavity ( $25 \mathrm{~mm}$ in diameter and $9 \mathrm{~mm}$ beneath surface) that was filled with a scattering solution (intralipid) and an absorbing dye (nirgosin) to simulate a tumor. At $658 \mathrm{~nm}$, the optical properties of the background were $\mu_{a-b k g}=0.0075 \mathrm{~mm}^{-1} \mu_{s-b k g}^{\prime}=0.89 \mathrm{~mm}^{-1}$, and the target optical properties were $\mu_{a-h e t}=0.033 \mathrm{~mm}^{-1} \mu_{s-h e t}^{\prime}=1.0 \mathrm{~mm}^{-1}$. The phantom system was modeled as before using the FEM forward model. Figure 1(b) demonstrates excellent agreement between DOS measurements of tumor phantom and FEM simulated linescans. Thus, the validity of the DOS measurement and the FEM simulation was established, and the failure of the FEM simulations to reproduce our previous clinical result in Fig. 1(a) suggests revision of our initial assumptions.

We believe that the discrepancy lies in the representation of the tumor as a discrete heterogeneity. We hypothesize that the spatial extent of the optical contrast is much larger than that of the US contrast. Such reasoning is not surprising because there is disagreement between radiological and histological size assessments between imaging modalities. ${ }^{9}$ The origins of contrast are inherently different for mammography, US, magnetic resonance imaging (MRI), and DOS. We refer to this as an "extended target" concept where the tumor is modeled as a Gaussian distribution of optical properties.

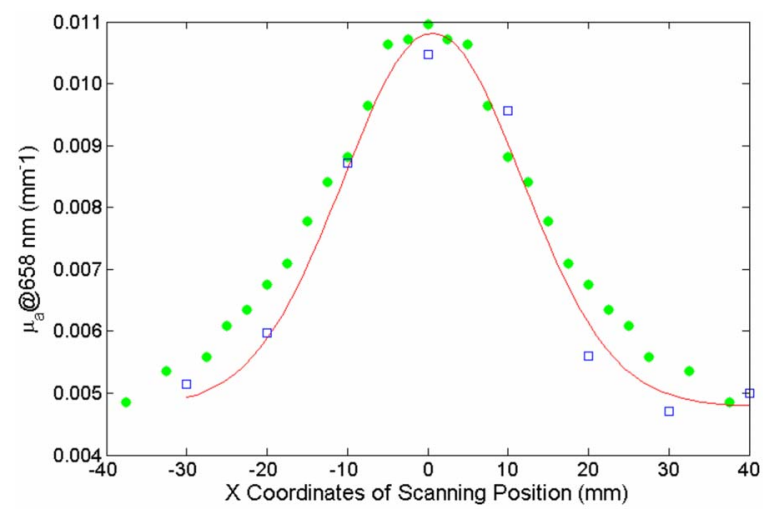

Fig. 2 Comparison of FEM forward simulation and DOS-measured linescans using the extended target concept. The squares and circles represent $\mu_{a-a v g}$ from measurement and simulation, respectively. The line is the Gaussian fit to the measured linescan. The tumor was modeled as a Gaussian distribution of optical properties with peak $\mu_{a} 5$ $\times$ the background, width of $25 \mathrm{~mm}$, and depth of $10.5 \mathrm{~mm}$.

To test this idea, we assumed that the clinical measurement reported a distributed peak value (i.e., $\mu_{a-h e t}$ ) that is $5 \times$ the background. For simplicity, we assumed only a distributed absorption coefficient. The center of the target distribution was the center of the lesion as defined by US, and the FWHM of the target optical property distribution was $25 \mathrm{~mm}$ to approximate the FWHM of the DOS linescan. The new simulated result with this extended target model showed much better agreement with experiment (i.e., Fig. 2). Other spatial distributions and contrast ratios may reproduce these observations. However, our main point is that the extended target concept implies that the absorption perturbation lies far beyond the conventional radiological target dimensions.

We retrospectively analyzed nine additional breast lesions (10 total) with various US depths and sizes; tissue pathology was confirmed by standard core biopsy. Five lesions were benign (i.e., fibroadenoma), and the remaining five were malignant (i.e., infiltrating ductal carcinoma). A summary of (benign versus malignant) tumor characteristics includes: average age (35 \pm 7.8 years versus $44.3 \pm 14.7$ years), US depth $(10.9 \pm 5.5 \mathrm{~mm}$ versus $15.8 \pm 5.3 \mathrm{~mm})$, and US maximum size $(13.0 \pm 6.0 \mathrm{~mm}$ versus $14.8 \pm 4.9 \mathrm{~mm})$. As before, the FWHM of the Gaussian fit to the DOS linescan data was significantly greater than the US dimensions $(21.1 \pm 4.5 \mathrm{~mm}$ for benign lesions and $29.0 \pm 9.7 \mathrm{~mm}$ for malignant lesions). High optical contrast (i.e., the ratio of measured $\mu_{a-a v g}$ to $\mu_{a-b k g}$ at $\left.658 \mathrm{~nm}\right)$ was observed for both benign (1.53 \pm 0.17$)$ and malignant $(2.06 \pm 0.4)$ lesions. In this small sample the $\mu_{a}$ contrast of malignant lesions was significantly higher than for benign lesions ( $z=0.02$, Wilcoxon Ranked Sum) despite that between groups, the lesions were comparable in size but the malignant lesions were deeper. The FWHM of the DOS linescan fit was approximately two-fold greater than the US maximum size for both benign and malignant tumors. We reproduced the clinical linescans in all 10 cases, as in Fig. 2. We conclude that our clinical observations are caused by distributed targets that are much larger than the size defined by anatomic imaging methods. The actual spatial extent of optical contrast is likely a realistic representation of tumor and normal tissue biology caused primarily by the spatially distributed presence of hemoglobin and water in the tumor, com- 


\section{JBO LETTERS}

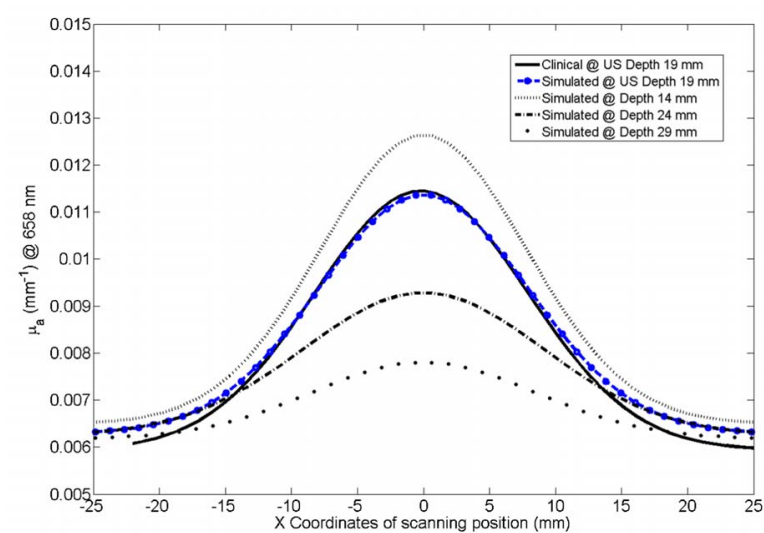

Fig. 3 The solid line is the Gaussian fit to the clinical linescan of another patient. (Individual measurement points not shown.) The dashed-dotted line is the simulated linescan with an extended target at a depth $19 \mathrm{~mm}$. The tumor was modeled as a Gaussian distribution of optical properties with peak absorption $5 \times$ the background and width $18 \mathrm{~mm}$. The remaining lines represent the forward simulation at different target depths $(14 \mathrm{~mm}, 24 \mathrm{~mm}$, and $29 \mathrm{~mm})$.

bined with the host tissue inflammatory response. ${ }^{10}$

The extended target concept can also evaluate tumor depth. We demonstrate depth sensitivity for an 11-mm-diam tumor (US dimension $11 \times 12 \times 12 \mathrm{~mm}$ at the depth of $19 \mathrm{~mm}$ ) in Fig. 3. The FWHM of the Gaussian fit to the DOS linescan data is $19 \mathrm{~mm}$. We plot simulated DOS linescans for distributed targets at a variety of depths. The peak $\mu_{a}$ of the distribution had $5 \times$ contrast versus background, as in the previous case. The target FWHM was close to the linescan FWHM $(18 \mathrm{~mm})$. We reproduced the clinical DOS linescan with our distributed target model only by using the US target depth (i.e., $19 \mathrm{~mm}$ ). We tried different contrast ratios in our simulations (at fixed 19-mm depth) and found that $3 \times$ and $4 \times$ contrast can also reproduce the clinical linescan, although the agreement was weaker. Similar agreement between simulated and actual DOS linescans was observed for all malignant tumors at the US depths. Another observation in Fig. 3 is that the target lesion would still be detectible even at a depth of $29 \mathrm{~mm}$ and a contrast of $5 \times$. Both $3 \times$ and $4 \times$ contrast ratios were also detectable. These results suggest that a $1-\mathrm{cm}$ tumor is detectable as deep as $29 \mathrm{~mm}$ using DOS reflectance. These results are highly dependent upon "physiological noise"; younger women have a higher background absorption, which lowers the effective contrast. If the normal fluctuation in $\mu_{a}$ is greater than the perturbation in $\mu_{a}$ from the target, the target will not be detected. Because spectral contrast is age dependent, multispectral approaches may help detect all breast lesions.

There are three major implications of our findings. First, one needs to be cautious when a priori information from other image modalities is used to constrain optical image reconstruction. Different imaging modalities are sensitive to different contrast mechanisms and tissue structures. Failure to properly consider the nature of the optical contrast may wrongly bias the optical reconstruction. The optical image contrast is inherently functional, whereas typically a priori information is structural. Second, the relatively low DOI spatial resolution may, under certain conditions, be offset because the physiological extent of the perturbation may exceed its anatomical dimensions. Thus the limiting factor of optical imaging is not necessarily spatial resolution, but "functional signal to noise." Our results show that relatively small $(\sim 1 \mathrm{~cm})$ stage I tumors can be detected at $\sim 3 \mathrm{~cm}$ in depth. This has important implications considering the difficulty of detecting 1-cm tumors in dense breast tissue and the excellent prognosis for patients who can begin early treatment. ${ }^{11}$ Third, image reconstruction based upon distributions may improve performance. By constraining the spatial distribution of optical properties, we also reduce the number of reconstructed variables dramatically, which eliminates regularization. In terms of instrument design, the larger spatial extent of the lesion relaxes the number of source-detector pairs necessary to visualize the target. This is attractive for DOS because of technical challenges in imaging with broadband spectra.

\section{Acknowledgments}

This work was supported by the National Institutes of Health under Grant Nos. P41-RR01192 (Laser Microbeam and Medical Program: LAMMP) and U54-CA105480 (Network for Translational Research in Optical Imaging: NTROI), the California Breast Cancer Research Program, and the Chao Family Comprehensive Cancer Center (P30-CA62203). BLI programmatic support from the Beckman Foundation and the Air Force Research Laboratory, under agreement number FA9550-04-1-0101 is acknowledged. The authors thank Montana Compton and Amanda F. Durkin for their assistance.

\section{References}

1. A. Cerussi, N. Shah, D. Hsiang, A. Durkin, J. Butler, and B. J. Tromberg, "In vivo absorption, scattering, and physiologic properties of 58 malignant breast tumors determined by broadband diffuse optical spectroscopy," J. Biomed. Opt. 11(4), 044005 (2006).

2. A. Li, G. Boverman, Y. Zhang, D. Brooks, E. L. Miller, M. E. Kilmer, Q. Zhang, E. M. Hillman, and D. A. Boas, "Optimal linear inverse solution with multiple priors in diffuse optical tomography," Appl. Opt. 44(10), 1948-1956 (2005).

3. B. Brooksby, S. Jiang, H. Dehghani, B. Pogue, K. Paulsen, C. Kogel, M. Doyley, J. Weaver, and S. Poplack, "Magnetic resonance-guided near-infrared tomography of the breast," Rev. Sci. Instrum. 75(12), 5262-5270 (2004).

4. Q. Zhu, S. H. Kurtzma, P. Hegde, S. Tannenbaum, M. Kane, M. Huang, N. G. Chen, B. Jagjivan, and K. Zarfos, "Utilizing optical tomography with ultrasound localization to image heterogeneous hemoglobin distribution in large breast cancers," Neoplasia 7(3), 263 270 (2005)

5. S. R. Arridge and J. C. Hebden, "Optical imaging in medicine. II. Modeling and reconstruction," Phys. Med. Biol. 42(5), 841-853 (1997).

6. A. Corlu, R. Choe, T. Durduran, K. Lee, M. Schweiger, S. R. Arridge, E. M. Hillman, and A. G. Yodh, "Diffuse optical tomography with spectral constraints and wavelength optimization," Appl. Opt. 44(11), 2082-2093 (2005).

7. A. H. Gandjbakhche, R. Nossal, and R. F. Bonner, "Resolution limits for optical transillumination of abnormalities deeply embedded in tissues," Med. Phys. 21(2), 185-191 (1994).

8. W. A. Wells, C. P. Daghlian, T. D. Tosteson, M. R. Grove, S. P. Poplack, S. Knowlton-Soho, and K. D. Paulsen, "Analysis of the microvasculature and tissue type ratios in normal versus benign and malignant breast tissue," Anal Quant Cytol. Histol. 26(3), 166-174 (2004).

9. P. T. Weatherall, G. G. Evans, G. J. Metzger, M. H. Saborrian, and A. M. Leitch, "MRI versus histologic measurement of breast cancer following chemotherapy: comparison with $\mathrm{x}$-ray mammography and palpation," J. Magn. Reson Imaging 13(6), 868-875 (2001).

10. C. K. Chow, "Imaging in inflammatory breast carcinoma," Breast Dis. 22(1), 45-54 (2005, 2006).

11. D. S. Buist, P. L. Porter, C. Lehman, S. H. Taplin, and E. White, "Factors contributing to mammography failure in woman aged 40-49 years," J. Natl. Cancer Inst. 96(19), 1432-1440 (2004). 